\title{
Autocuidado en el manejo de pleurovac y ejercicio respiratorio en pacientes de cirugía de hombres del Hospital Dr. Mario Catarino Rivas
}

\author{
Santa Lizeth Sánchez ${ }^{1}$ \\ Emelda Ruth Suazo ${ }^{2}$
}

\section{RESUMEN}

La sala de cirugía de hombres, de cirugía general, tiene una capacidad de 20 pacientes, en la cual se observa un el alto índice de pacientes ingresados a este servicio por herida de arma de fuego 0 arma blanca, en edades comprendidas entre los 14 y 28 años de edad.

El alto índice de violencia que sufre el país, provoca que se sobrepase la capacidad de este servicio con pacientes con trauma torácico, los cuales son manejados con sistema de drenaje torácico: sello de agua (pleurovac). El propósito de este sistema es ayudar al pulmón a expandirse y extraer el líquido que se encuentre en él provocado por el trauma.

Pero, al momento de su manejo, se ha observado que el paciente no está instruido sobre la forma de manipular el sistema de drenaje (pleurovac). Con respecto, al personal auxiliar de enfermería, se observa que no dan la importancia que tiene a orientar al paciente sobre su manejo y la realización de ejercicios respiratorios. En consecuencia, se espera que con esta investigación-acción se le dé importancia a la necesidad de fortalecer el conocimiento del personal auxiliar de enfermería sobre este tema, lo cual impulsará un proceso de gestión de autocuidado en el manejo de pleurovac y ejercicios respiratorios para favorecer la atención de calidad al usuario, asegurando las dimensiones de continuidad y eficacia.

Palabras clave: autocuidado, pleurovac, ejercicio respiratorio, trauma torácico.

${ }^{1}$ Estudiante de Enfermería, Departamento Ciencias de la Enfermería, Escuela Universitaria de Ciencias de la Salud, UNAH-VS; dirección electrónica: santalizethsanchezsantos@yahoo.com

2 Docente de Enfermería, Departamento Ciencias de la Enfermería, Escuela Universitaria de Ciencias de la Salud, UNAH-VS; dirección electrónica: emeldaruthsuazo@yahoo.com 


\section{ABSTRACT}

The men's operating room has a capacity of 20 patients in general surgery, which shows that the high rate of patients admitted to this service are due to firearm injury or white weapon and their age is from 14 to 28 years. The high rate of violence exceeds the capacity of this service attending patients with thoracic trauma which are treated with chest drainage system (pleurovac). The purpose of this system is to help the lung to expand and remove fluid that is found in the trauma. But at the time of its management, it has been observed that the patient is not well trained on how to use the drainage system (pleurovac). With respect to the auxiliary nursing staff it is observed that they do not realize how important it is to guide the patients during their respiratory exercises. With this study, we intend to strengthen the auxiliary nursing staff's knowledge to promote a self-care management process in handling pleurovac and breathing exercises to promote continuity and efficiency dimensions of human quality care.

Key words: pleurovac, breathing exercise, self-care, thoracic trauma.

\section{INTRODUCCIÓN}

La enfermería es la ciencia del cuidado de la salud del ser humano, que además proporciona a las personas y grupos asistencia directa en su autocuidado, según sus requerimientos, debido a las incapacidades que vienen dadas por sus situaciones personales.

La salud se define como el estado de la persona que se caracteriza por la firmeza o totalidad del desarrollo de las estructuras humanas y de la función física y mental, por lo cual la salud es un concepto inseparable de factores físicos, psicológicos, interpersonales y sociales. Se refiere también a la promoción y el mantenimiento de la salud, el tratamiento de la enfermedad y la prevención, que se han establecido entre los conceptos de persona, entorno y salud, si bien se incluye en este concepto meta paradigma el objetivo de la disciplina, que es "ayudar a las personas a cubrir las demandas de autocuidado terapéutico o a cubrir las demandas de autocuidado terapéutico de otros".

Al respecto, se pueden utilizar métodos de ayuda en cada uno de los sistemas de enfermería, puesto que cuidar a los pacientes es un arte y una ciencia que exige una formación formal y el papel de la enfermera es poner al sujeto en las mejores condiciones para que la naturaleza actúe sobre él. El concepto se amplía considerando su contexto, para comprender y explicar la situación de salud 
individual y colectiva. El cuidado se lleva a cabo para contribuir a recuperar la salud, prevenir la enfermedad, fomentar el mantenimiento de la salud integral de la persona, familia y comunidad.

Esta investigación-acción es prospectiva y transversal. Se recolectó la información entre los meses de febrero a marzo de 2011, implementando un proceso de gestión en el autocuidado de manejo de pleurovac y ejercicio respiratorio entre los meses de abril y mayo de 2011.

El universo y la muestra de la investigación están conformadas por los pacientes con patología de trauma torácico que se les coloca un tubo endopleural y los familiares de estos, junto con el personal auxiliar de enfermería que presta este servicio.

La metodología empleada en la investigación-acción fue la observación participativa, aplicando la encuesta y la entrevista, para la recolección de datos y ejecución de la misma. Para las intervenciones educativas con pacientes y familiares se aprovechó a los estudiantes de enfermería y se asignó al personal de turno brindar educación a pacientes con pleurovac y para el personal auxiliar de enfermería se planificaron reuniones los días viernes de cada fin de mes. Asimismo, se realizaron jornadas educativas sobre el tema con los camilleros de recuperación y de emergencia de cirugía, quienes son los encargados de movilizar a estos pacientes.

\section{Escenario y participantes}

Esta investigación-acción se realizó en el Hospital Dr. Mario Catarino Rivas y se aplicó a los pacientes ingresados a la sala de cirugía de hombres con un trauma torácico y presencia de tubo endopleural. Tales personas llegaron de otros hospitales, áreas rurales y áreas urbanas de San Pedro Sula. La mayoría no tiene conocimiento sobre su patología o del cuidado que debe tener al momento de usar un tubo endopleural o de los ejercicios que le ayudarán a expandir el pulmón.

Es posible señalar que se observa un alza de pacientes en temporadas festivas o de feriados, tales como Semana Santa, feria de junio, mes de diciembre o en los encuentros deportivos de futbol; ya que en estos es cuando la población se expone a la ingesta excesiva de bebidas alcohólicas y uso de drogas (ver gráfico 1). 
Gráfico 1. Origen del trauma en los pacientes

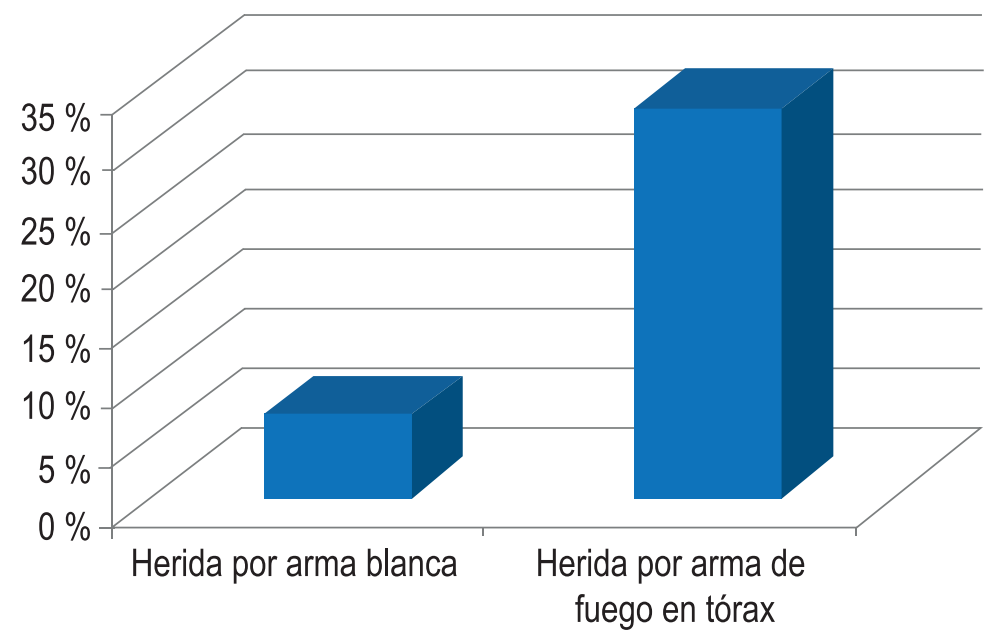

\section{RESULTADOS}

Según lo explicado por el personal auxiliar de enfermería, al ingresar el paciente se realiza la actividad educativa de explicar a los pacientes sobre los riesgos de tener un tubo endopleural. Al preguntarle si realizan la medición de la producción del drenaje, responden que lo hacen una vez al día, pero se observa que no la cuantifican de manera adecuada, ni realizan el registro en la hoja de ingesta y excreta del expediente.

Después, al preguntarle si realizan la educación sobre ejercicios respiratorios manifiestan que los realizan en un $100 \%$. Sin embargo, al preguntar a los pacientes sobre el manejo del pleurovac, el 80 \% contestó que al recibir la educación ellos se encontraban con dolor y, por lo tanto, no prestaron la atención necesaria (ver gráfico 2).

Gráfico 2. Orientación sobre el manejo del pleurovac

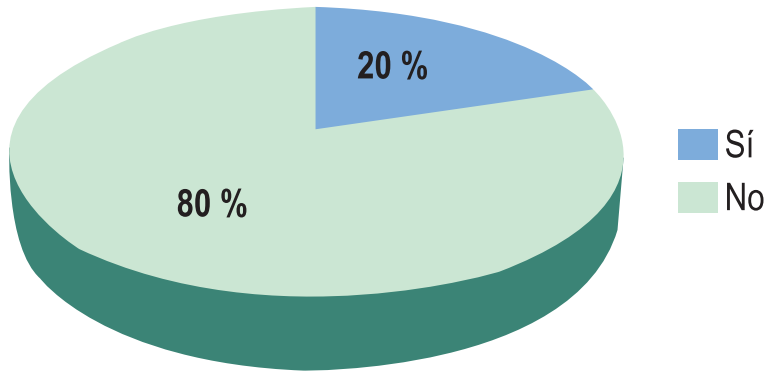


El $70 \%$ de los pacientes entrevistados contestó que sí saben cómo se realizan los ejercicios respiratorios, aunque se observó que estos realizan los ejercicios solo ante la presencia del personal auxiliar. Además, se encontró que se utilizan espirómetros improvisados con guantes y el paciente no adquiere la postura adecuada para realizar dichos ejercicios (ver gráfico 3).

Un $40 \%$ de los pacientes desconocen las complicaciones de dejar caer el pleurovac o de sacarse el tubo endopleural al momento de movilizarse con el pleurovac; lo cual se convierte en una amenaza para la vida de estos pacientes.

Gráfico 3. Práctica de los ejercicios respiratorios

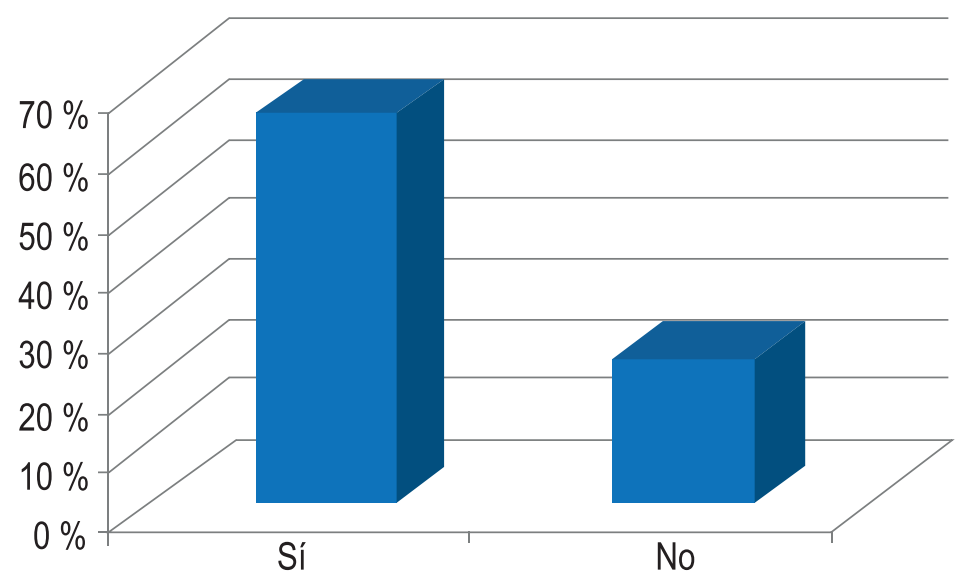

\section{CONCLUSIONES}

La actividad educativa que hasta este momento se ha estado realizando por el personal auxiliar de enfermería, ha sido de forma incidental, por lo que se debe retomar la educación continua en el personal, para que ellos mejoren la atención que brindan.

Esta investigación-acción proveyó de los insumos necesarios para impulsar una estrategia de motivación hacia la educación permanente durante el ingreso, estancia y egreso del paciente, lo cual ayudará a reducir los días de estancia y el aumento de cobertura de antibióticos a estos pacientes.

Se propone programar capacitaciones al personal de servicio, elaborar material de consulta y trifolio educativo, entre otras acciones. Asimismo, se recomienda 
implementar intervenciones educativas permanentes con los estudiantes que realizan sus prácticas clínicas e involucrar a las familias de los pacientes en las actividades educativas.

\section{AGRADECIMIENTO}

Al personal que trabaja en el área de cirugía de hombres en el Hospital Dr. Mario Catarino Rivas, en particular a la jefa de Enfermería del servicio, licenciada Lourdes Sauceda.

\section{BIBLIOGRAFÍA}

Costa Mendes, I.A. (2005). Enfocadas en el cuidado de enfermería. Ciencias y Enfermería, (XI), 2, pp. 35-46.

Estefo Agüero, S. (2010). Enfermería en el rol de gestora de los cuidados. Ciencias y Enfermería, (3), XVI, pp. 33-39.

Montesinos Jiménez, G. (2002). El conocimiento y la enfermería. Revista Mexicana de Enfermería Cardiológica, (10), 1, pp. 4.

Potter, PerryA. (2002). Fundamentos de enfermería. St. Louis: Elsevier Mosby.

Zárate, R. (2004). La gestión del cuidado de enfermería. Index Enferm, (13), pp. 4445. 